\title{
Training for fostering knowledge co-construction from collaborative inference-drawing
}

\section{Journal Article}

\section{Author(s):}

Deiglmayr, Anne; Spada, Hans

Publication date:

2011-06

Permanent link:

https://doi.org/10.3929/ethz-b-000130818

\section{Rights / license:}

Creative Commons Attribution-NonCommercial-NoDerivatives 4.0 International

\section{Originally published in:}

Learning and Instruction 21(3), https://doi.org/10.1016/j.learninstruc.2010.06.004 
Running head: Training for collaborative inference-drawing

\title{
Training for fostering knowledge co-construction from collaborative inference-drawing
}

\author{
Anne Deiglmayr *, Hans Spada \\ Institute of Psychology, University of Freiburg, 79085 Freiburg, Germany
}

\begin{abstract}
Groups typically have difficulties drawing inferences that integrate individuals' unique information (collaborative inferences) and thus yield a true assembly bonus. An experiment with 36 dyads of university-level students in four training conditions showed, particularly in untrained dyads, that collaborative inferences were less likely to be drawn than two other types of inferences that did not require collaborative information processing. Reflected case-based training led to more inferences during subsequent testing. Testing performance was highest if the training had also included explicit strategy instruction in combination with adaptive tutoring. However, explicit strategy instruction without adaptive tutoring offered no advantage.
\end{abstract}

Keywords: Collaborative problem-solving; Inference drawing; Tutoring; Collaboration strategies

* Corresponding author. Tel.: +49 761 2039163; fax: +49 7612032490

E-mail addresses: deiglmayr@psychologie.uni-freiburg.de (A. Deiglmayr) \& spada@psychologie.uni-freiburg.de (H. Spada)

This is an author's version, published under a Creative Commons Attribution Non-Commercial No Derivatives License

The published version of this article can be found at doi.org/10.1016/j.learninstruc.2010.06.004

Please cite as:

Deiglmayr, A., \& Spada, H. (2011). Training for fostering knowledge coconstruction from collaborative inference-drawing. Learning and Instruction, 21(3), 441-451. 


\section{Introduction}

Solving problems in collaboration with others is a very effective way of learning. In fact, collaborative learning activities in which students build upon one another's contributions and actively co-construct ideas, explanations, and solutions have the potential to be more effective than even the most constructive individual learning activities (Chi, 2009). This is particularly the case if learners complement one another in their knowledge or perspectives (Aronson, Balney, Stephan, Sikes, \& Snapp, 1987; Webb \& Palincsar, 1996), because collaborators then have the chance to co-construct knowledge and derive solutions that no individual person could have created alone (Kneser \& Ploetzner, 2001; Rummel \& Spada, 2005). A central activity for such knowledge co-construction is the drawing of collaborative inferences (Deiglmayr \& Spada, 2010a).

Inference drawing is very important for learning and reasoning because inferences establish meaningful connections that enable knowledge integration, deeper understanding of learning materials, and better solutions. For example, inferences are essential in the comprehension of discourse and texts (Black, Freeman, \& Johnson-Laird, 1986; Kintsch, 1998), when integrating information from different representations such as text and pictures (Bodemer, Ploetzner, Feuerlein, \& Spada, 2004; Schnotz \& Bannert, 2003), or when synthesizing information from different sources as, for example, multiple documents (Pennington \& Hastie, 1993; Strømsø, Bråten, \& Britt, 2010). In the present study, the focus is on the inferences people make when trying to come up with a plausible explanation (i.e., plausible inferences; Collins \& Michalski, 1989). These inferences combine (at least) two pieces of information and, on the background of general knowledge, transform them into a new piece of information. However, contrary to most existing accounts of the role of inferences in reasoning, in the present study the focus is not on individual information-processing but rather on the co-construction of knowledge between two collaborating partners.

Collaboratively drawn inferences are important for effective collaborative problem-solving, facilitate the co-construction of explanations, and thus foster learning (Chi, 2009; Kaartinen \& Kumpulainen, 2002). There are even situations in which collaboratively drawn inferences are the only way of arriving at important conclusions. This is the case if the information enabling the inferences are distributed between students, for example due to students' complementary training and expertise (Rummel \& Spada, 2005), or as a result of learning arrangements designed to create resource interdependence, such as the jigsaw method (Aronson et al., 1987). In these situations, the benefits of collaborative inferences are most visible. As an illustration, consider a student in clinical psychology and a medical student who are developing a therapy plan for a patient as part of a collaborative learning exercise on comorbid mental and medical pathology (cf. Rummel \& Spada, 2005). The students' patient has been diagnosed with a moderate episode of major depression in combination with an acute relapse of multiple sclerosis. To set up an effective treatment plan, the students now need to exchange and integrate their complementary expertise on the two disorders. For example, part of the psychology student's (but not the medical student's) knowledge about effective treatments for depression is the information that cognitive therapy, in which the patient learns to restructure dysfunctional negative thoughts, has been proven to be a very 
effective method for treating mild to moderate depressive episodes. Part of the medical student's (but not the psychology student's) knowledge about multiple sclerosis, on the other hand, is the information that multiple sclerosis often leads to severe restrictions in attention and working memory resources. If the students exchange these two pieces of information, they can collaboratively draw the inference that patients with multiple sclerosis may not be able to benefit from cognitive psychotherapy due to their cognitive restrictions. As a result, the students can set up a more suitable therapy plan for their patient, for instance, by proposing medication with antidepressants and more behaviourally oriented psychotherapy.

Previous research has shown that even university-level students often have substantial difficulties drawing collaborative inferences and thus making optimal use of their complementary knowledge resources (Deiglmayr \& Spada, 2010a; Härder, 2003; Rummel \& Spada, 2005). The experiment reported in this article, therefore, aimed to increase the collaboration skills of university-level students by training them in the application of specific collaboration strategies designed to promote collaborative inferences. In addition, collaborative inferences were compared to two other inference types that did not require collaboration.

\subsection{Studying collaborative inference-drawing experimentally}

To study the drawing of collaborative inferences under controlled conditions, we built on the information pooling paradigm from the social psychology literature on decision making in small groups (Stasser \& Titus, 1985). The information pooling paradigm typically uses decision tasks with one optimal and several suboptimal decision alternatives, such as choosing the most suitable candidate for a job. A pre-defined number of critical information items are distributed between group members according to a specific scheme (called a "hidden profile") so that the optimal decision only becomes apparent if group members pool their unique, or "unshared" information (Wittenbaum, Hollingshead, \& Botero, 2004). Because the distribution of critical information is manipulated experimentally, and thus known to the experimenter, the proportion of their unshared information each person actually contributes during group discussion can be established.

While information items are treated as being independent of one another in the classical information pooling paradigm (Fraidin, 2004), our adapted version looks at pairs of interdependent pieces of information, that is, the premises of solution-relevant inferences. For example, one of the tasks that we also use in the present experiment is a fictitious murder-mystery in which students have to find the guilty person among four suspects (cf. Deiglmayr \& Spada, 2010a). Two students first study two systematically different sets of "interrogation protocols" individually before they discuss the case and agree on a joint solution. Incriminating evidence against the true murderer, and thus the correct solution, has to be inferred from pieces of information that are themselves neither very incriminating nor very exonerating. For example, while the guilty suspect claims to have been in bed and unable to sleep because of an argument in the adjacent room, a witness states that this argument ended long before the time of the murder. Thus, it can be inferred that the suspect has been lying to the detective in order to fabricate an alibi. Twelve information pairs yielding twelve solution-relevant inferences are distributed 
between the two students. In this way, it is possible to establish the proportion of possible inferences students actually draw during their problem solving. In addition, by experimentally manipulating the distribution of critical information between students, collaborative inferences can be systematically compared to inferences that do not require collaboration.

Depending on the way in which two interdependent pieces of information (symbolized by two complementary puzzle pieces in Figure 1) are distributed in the dyad, three types of inferences can be distinguished, namely collaborative, individual, and shared (Deiglmayr \& Spada, 2010a). These inference types are manipulated experimentally by distributing information to students in specific ways and thus are defined a priori. Information is either known to both students (shared) or known to only one student (unshared); two complementary pieces of unshared information are either located with the same person (undistributed) or with two different persons (distributed). Collaborative inferences result from unshared information that is distributed between collaborators; thus, the inference can only be drawn collaboratively. Individual inferences, on the other hand, result from unshared information that is located with the same person. Thus, they can be drawn individually without the need for collaboration. Shared inferences, finally, result from shared information. They can be drawn individually by one or both partners, or collaboratively between them. Prior research (Deiglmayr \& Spada, 2010a; Härder, 2003; see also Fraidin, 2004) has shown that collaborative inferences have the lowest probabiliy of actually being drawn, while individual inferences have a higher probability and shared inferences have the highest of all. In a previous study (Deiglmayr \& Spada, 2010a), for example, dyads solving the murder mystery task drew on average $49 \%$ of the possible collaborative inferences, $65 \%$ of the possible individual inferences, and $79 \%$ of the possible shared inferences.

Insert Figure 1 about here

\subsection{Training for drawing collaborative inferences}

The main aim of the present study was to improve students' skills for drawing collaborative inferences. The training developed for this purpose involved the collaboration on a carefully designed training problem (diagnosing a patient with one out of several fictitious tropical diseases), followed by a collaborative reflection phase. Further, the experiment tested the incremental effectiveness of two additional training interventions, namely explicit instruction in several strategies for drawing collaborative inferences, and adaptive guidance from a semi-computerized tutoring tool. As a result of training, students were expected to be able to transfer their newly acquired collaboration skills to a subsequent case-based testing phase. The testing phase used a structurally similar problem-solving task, but from a very different task domain (i.e., solving a murder mystery).

\subsubsection{Reflected case-based training}

A difficult collaborative problem-solving task served as the training case. Students had to find the correct diagnosis for a patient. Their individual information as 
well as most individual and shared inferences favoured an incorrect diagnosis, while the correct diagnosis could only be found by drawing multiple collaborative inferences. After their own collaborative solution attempt, students were given the correct diagnosis including the most important inferences leading towards it. During a subsequent collaborative reflection phase they were prompted to reflect on their collaboration and discuss how they might improve their collaboration on the upcoming testing case. Research on "learning by doing", for example, in goal-based learning scenarios (Schank, Berman, \& Macpherson, 1999) or problem-based learning (Dochy, Segers, Van den Bossche, \& Gijbels, 2003), indicates that students can acquire relevant problem-solving and collaboration strategies implicitly from specifically designed training cases. Transferable acquisition of problem-solving and collaboration strategies can be greatly improved by deliberately reflecting on one's past performance (Cohen, 1994). For example, the so-called "group-processing technique" helps students develop transferable collaboration skills by having them discuss, aided by teacher feedback, what specific member behaviours were helpful or unhelpful for solving a joint problem, and decide what they want to do similarly or differently in future collaboration (Johnson \& Johnson, 1993). Thus, it was expected that reflected case-based training would allow students to improve their skills for drawing collaborative inferences, and thus lead to better performance during subsequent testing.

\subsubsection{Explicit strategy instruction}

In two of the experimental conditions, reflected case-based training was coupled with explicit strategy instruction aimed to inform students about three collaboration strategies. As a general inference-drawing strategy, students were instructed to look for interconnections between incoming information (e.g., while listening to a partner) and old information (i.e., information learned while studying the problem individually) that allowed for solution-relevant inferences to be drawn (information connection strategy). Over and beyond the search for connections, collaborative inferences additionally require effective communication between students (Deiglmayr \& Spada, 2010a). First, to enable a collaborative inference, a speaker has to pool a piece of yet unconnected information. The speaker cannot yet relate that information to a possible problem solution; such an inference would have to be drawn collaboratively and require information only known to his or her partner. To make more efficient use of their unshared information, students were therefore told to keep in mind that their own information might take on new meaning and relevance when considered in the light of what their partner knew about the problem. Consequently, they were instructed to inform their partner not only about what they knew to be relevant for finding a solution, but also about information they could not yet fit into the bigger picture (information pooling strategy). Second, to draw a collaborative inference once a speaker has pooled a piece of unshared information, the listener must take up that new information and integrate it with his or her own knowledge. Students were therefore instructed to focus their reactions on the new information in their partner's contributions, rather than, for example, to try and confirm the shared information they already knew themselves (information take-up strategy). Information about these three strategies was compiled in a short text (described in more detail in the Method section) that students read prior to their collaboration on the training case. Even without such explicit instruction, reflected practice with carefully designed 
training problems can lead to improved problem-solving and collaboration skills. However, students typically benefit from additional instruction in which relevant problem-solving steps and behaviours are explicitly described and/or demonstrated to them (Dansereau et al., 1979; Rummel \& Spada, 2005; Star \& Rittle-Johnson, 2008; Zohar \& Peled, 2008). Thus, in the present study, it was expected that providing explicit strategy instructions to students would increase the effectiveness of the training phase.

\subsubsection{Adaptive tutoring}

Successful training for problem-solving and collaboration strategies often employs not only explicit instruction on what students are supposed to do, but also offers guidance through immediate feedback and adaptive prompts (Deiglmayr \& Spada, 2010b). Some of the most impressive demonstrations of the effectiveness of such "coached" problem-solving come from human tutors as well as computer-based intelligent tutoring systems (Anderson, Corbett, Koedinger, \& Pelletier, 1995; VanLehn et al., 2007). For training in the present study a semi-computerized inference tutoring tool was developed, which provided students with just-in-time feedback on their performance, pointed out relevant cues (e.g., new information being mentioned by a student), and prompted strategy application (e.g., take up and integrate new information). The tool is described in detail in the Method section. Guidance from the inference tutoring tool was provided during the training phase only, where it aimed to help students apply the strategies they had just been instructed to use. Accordingly, it was expected that the tool would increase the effectiveness of the explicit strategy instruction.

\subsection{The present study - Hypotheses}

Developing an effective training for collaborative inference-drawing was the main aim of the present study. Four different training conditions were tested: (a) the basic training condition, (b) the instructed training condition, (c) the tutored training condition, and (d) a control condition that received no training at all. Moreover, three types of inferences were examined: (a) collaborative inferences, (b) individual inferences, and (c) shared inferences. It was expected that the trained conditions would outperform the control condition (Hypothesis 1a). Further, differences between the three training conditions were expected in the sense that an increase in support during training would lead to better performance during testing. First, explicit strategy instruction prior to solving the training case (instructed training) was expected to be more effective than solving the training case without explicit strategy instruction (basic training; Hypothesis 1b). Second, instructed training in combination with adaptive tutoring during collaboration on the training case was expected to yield the best training results as compared to basic and instructed training (Hypothesis 1c).

A further aim of the present study was to investigate the differential difficulty of drawing collaborative, individual, and shared inferences. In line with previous findings (Deiglmayr \& Spada, 2010a), the inference-type hypothesis was formulated, namely that collaborative inferences would be less likely to be drawn than individual inferences (Hypothesis 2a) and that both collaborative and individual inferences would be less likely to be drawn than shared inferences (Hypothesis 2b). Further, an interaction between kind of training and inference type was expected, that is, the effect of inference type should be 
most pronounced in the control condition, and decline with the positive effects of training (Hypothesis 2c).

\section{Method}

\subsection{Design}

All training interventions were administered during the training phase in which students worked on the medical-diagnosis case. Training effects were assessed during the testing phase in which students worked on the murder-mystery case (see Figure 2).

Insert Figure 2 about here

The experimental design was 4(kind of training: control / basic training / instructed training / tutored training) x 3(inference type: collaborative / individual / shared) with inference type as repeated measures, that is, within-dyads factor. An untrained control condition was compared to the three trained conditions, with each condition realizing one additional training component; that is, basic training dyads engaged in reflected case-based training, instructed training dyads were additionally given explicit strategy instruction, and tutored training dyads were further helped by the inference tutoring tool during the training phase. During the testing phase students were neither explicitly instructed on how to collaborate, nor did they have access to the materials and tools that had been available during the training phase.

Inference type was manipulated during both training and testing by systematically distributing case information between the two students. In the training phase, each dyad encountered the same version the medical-diagnosis training case that had been constructed to maximize the importance of collaborative inferences for finding the correct solution. In the testing phase, three counterbalanced text versions of the murdermystery problem were used in order to correct for the possibility of confounding effects of inference type with effects of inference content. Thus, whether the two interdependent pieces of information yielding a solution-relevant inference were distributed between students (requiring a collaborative inference; cf. Figure 1), located with the same student (individual inference), or provided to both students (shared inference) depended on the specific text version. However, information distribution in all text versions ensured that every dyad could draw four collaborative, four individual, and four shared inferences.

\subsection{Participants}

Participants were 72 female university students (mean age $=22.4$ years, $\underline{\mathrm{SD}}=$ 2.49), majoring in various subjects. Psychology and medical students were excluded to reduce heterogeneity in relevant prior knowledge. All participants were native speakers of the German language in which the experiment was conducted. Only students who did not know each other were assigned to the same dyad. The resulting 36 dyads were randomly assigned to the four conditions, each condition containing 9 dyads.

\subsection{Material}




\subsubsection{The training case}

The training case materials provided students with a written description of the symptoms, anamnesis, and travel itinerary of a patient who had just returned from a vacation in the tropics, as well as the main symptoms, pathogenesis, and prevalence of three (fictitious) tropical diseases. The dyads' task was to diagnose the patient with the correct disease. Each student read a systematically different set of information prior to collaboration, and all texts had to be returned before students were allowed to communicate. Information was distributed in such a way that each dyad had to draw collaborative, individual, and shared inferences. However, collaborative inferences were particularly important for finding the correct diagnosis. For example, one student knew that there was a "crane flue" epidemic in the countries neighbouring Mangrovia- the tropical paradise the patient had visited during his vacation - but no case of crane flue in Mangrovia itself. Her partner, on the other hand, knew that the patient had not only visited Mangrovia, but had also spent some days exploring one of its neighbouring countries. Thus, students could draw the collaborative inference that the patient might have contracted the crane flue disease (which was, in fact, the correct diagnosis).

\subsubsection{The testing case}

The murder-mystery problem serving as the testing case was adopted with small modifications from a previous study (Deiglmayr \& Spada, 2010a). The case was presented to participants in the form of written "interrogation protocols", that is, interviews with suspects and witnesses, and a description of the crime scene. In total, the text contained 24 solution-relevant pieces of case information, embedded in a larger story. The dyad's task was to find a murderer among four suspects by determining motives, checking alibis, and finding additional pieces of evidence linking the suspects to the crime. Every dyad could draw 12 inferences (four collaborative, four individual, and four shared), yielding a motive, an alibi, and a piece of evidence for each suspect. As in the medical-diagnosis problem, each student studied a systematically different set of information prior to collaboration, and all texts had to be returned before students were allowed to communicate. ${ }^{1}$

\subsubsection{Questionnaires}

When entering the experiment's participant pool, students filled in a questionnaire asking for their experience with computer use (frequency ratings for use of word processing software, email, chat, and the internet).

After finishing their collaboration on the testing case, students individually filled in a questionnaire asking for their age, their final academic grade from high-school (the German "Abitur"), and the subject they were majoring in. In addition, students stated their experience with murder-mystery stories and films (estimated number of hours spent reading/watching during a typical week) and their knowledge about tropical diseases (prior exposure to topic, yes or no).

\footnotetext{
${ }^{1}$ The complete, original case materials can be obtained from the first author, or downloaded from http://www4.psychologie.uni-freiburg.de/signatures/ameier/
} 


\subsection{Procedure}

\subsubsection{Basic training}

The Basic Training dyads collaborated on the medical-diagnosis problem without receiving explicit instructions concerning their collaboration. Afterwards, they engaged in the collaborative reflection phase. In this phase, students received a model solution that stated the correct diagnosis and explained important inferences leading towards it. Students were prompted to discuss the model solution, evaluate their own collaboration on the case, and think of ways in which they might be able to collaborate "even better" on the upcoming murder-mystery problem (a short description of that task was provided).

\subsubsection{Instructed training}

The Instructed Training dyads, in addition to the basic training, received explicit strategy instruction prior to their collaboration on the training case. An expository text of approximately 850 words, compiled in a thin booklet, introduced students to the information connection, the information pooling, and the information take-up strategies (see section 3.1). It explained which problem each strategy addressed (e.g., "While new information is particularly important, it is also often overlooked during group discussions"); it also explained how this strategy was relevant for the medical-diagnosis problem (e.g., "You will learn new information regarding the diseases and the patient from your partner; this new information is particularly important for making a good decision"), and detailed what students were expected to do. For example:

(...) Pay special attention to all new pieces of information [you learn from your partner] even if they do not seem very important to you at first sight. Take your time to reflect on that information, and try to match information on a disease with information on the patient, and the other way round. It may be a good idea to signal to your partner that a piece of information is new to you, so she can tell you more about it.

Students were asked to practice the strategies during their collaboration on the training case, and were prompted to reflect on their strategy application during group processing.

\subsubsection{Tutored training}

The Tutored Training dyads, in addition to receiving instructed training as described above, were assisted by a semi-computerized inference tutoring tool. The tool provided adaptive feedback and prompts during students' collaboration on the training case. It was controlled by a human observer who followed collaborators' discussions of the medical-diagnosis problem and identified when solution-relevant pieces of patient or disease information were mentioned and solution-relevant inferences drawn. The observer filled in an observation matrix and sent four types of messages to students via the tutoring tool, based on a developing graphical representation of inference patterns in the observation matrix (see Figure 3).

Insert Figure 3 about here 
Whenever a piece of case information was mentioned for the first time, new information feedback was given. Collaborators heard a specific sound and saw the following message:

"New information! Complementary information is located with \{Name of Person A\} / \{Name of Person B $\}$ / both of you."

This feedback facilitated the application of the information take-up strategy by drawing students' attention to the new information, and of the information connection strategy by indicating the owner of complementary information. In addition, it provided positive feedback for successful information pooling. Whenever a solution-relevant inference was drawn, complete inference feedback was given, which informed students about the correctness of their inference and provided positive feedback for successful information connection ("Well done! You have just discovered an important connection.").

Towards the end of their discussion, students also received reminders and hints to make sure that they had the opportunity to practice inference drawing even if they had not yet pooled important pieces of patient or disease information. First, students received a missing information reminder for each incomplete inference, that is, a short summary of a piece of information already discussed but not yet integrated by an inference, plus a prompt to search for complementary information. In cases where neither the patient information nor the disease information enabling a specific inference had been discussed, or if collaborators could not supply missing information despite prompting, a missing information hint provided a short summary of the missing information.

\subsection{Data coding - Measures}

Collaborators' discussions of the murder-mystery problem in the testing phase were transcribed and the transcripts analyzed by a trained coder who was blind to both training condition and inference type. The coder determined for each of the 24 pieces of text information and for each of the 12 inferences whether it had been mentioned or not. Inter-coder reliability was established with the help of a second person who coded one third of the videos (agreement for text information: 97.9\%; Cohen's kappa $=.87$; agreement for inferences: $90.3 \%$; Cohen's kappa $=.71$ ). The proportion of collaborative, individual, and shared inferences that were actually drawn during discussion (inference drawing frequency) was the most important dependent variable for our analyses. In addition, we also calculated the proportion of shared, unshared undistributed, and unshared distributed pieces of text information that were mentioned during discussion (information pooling frequency). Solution correctness, that is, whether a dyad chose the correct suspect in the murder-mystery problem, served as an outcome measure.

\subsection{Setting}

During the whole experiment, except for a face-to-face introduction phase and a short recreational break between training and testing phase, collaborators sat in adjacent rooms. During phases of individual work they could neither see nor hear their partner. During collaborative phases they could talk to each other over an audio connection 
(headset). In addition, they had access to a shared word processor. The computermediated collaboration setting established controlled conditions in which all utterances and actions could be easily observed and recorded. The observer operating the inference tutoring tool sat in a third room and remotely monitored the ongoing discussion; the tutor messages were simultaneously displayed in both participants' rooms. Students' discussions in the training and testing phase as well as their computer screens were videotaped for later analysis.

\section{Results}

\subsection{Confounding variables}

ANOVAs over the questionnaire data confirmed that participants assigned to the four conditions did not differ in their age, $\underline{F}(3,71)=1.02, \underline{p}=.39$; in their academic grades, $\mathrm{F}(3,71)=.87, \underline{\mathrm{p}}=.46$; in their frequency of computer use (word processing software $), \underline{F}(3,70)=1.27, \underline{p}=.29$; in their frequency of e-mail use, $F(3,70)=.66, \underline{p}=$ .58 ; in their frequency of chat use, $\mathrm{F}(3,70)=.49, \mathrm{p}=.69$; in their frequency of internet use, $\underline{\mathrm{F}}(3,70)=.37, \underline{\mathrm{p}}=.77$, or in their typical weekly number of hours exposed to murder-mystery stories and films, $\underline{\mathrm{F}}(3,71)=.74, \underline{\mathrm{p}}=.53$. Conditions also did not differ with regard to the number of participants with and without prior exposure to the topic of tropical diseases, $\chi^{2}(2, \underline{N}=72)=1.87, \underline{p}=.39$.

\subsection{Manipulation check (Training phase)}

Inspection of the videotapes confirmed that all dyads followed instructions during the training phase; in particular, all dyads discussed their collaboration on the training case and made plans for improving their collaboration on the testing case during the reflection phase. Dialog analyses (content analysis) confirmed that the combination of all three training components (i.e., tutored training) led to near optimal performance during training, that is, basic training and instructed training dyads showed the familiar pattern of drawing collaborative < individual < shared inferences, whereas tutored training dyads drew nearly all possible inferences of all three inference types (see Table 1).

Accordingly, a two-way mixed-factor ANOVA revealed significant main effects for both the within subjects factor Inference Type, $\underline{F}(2,48)=44.78, \underline{p}<.01$, partial $\eta^{2}=$ .65 , and the between subjects factor Kind of Training, $\underline{F}(2,24)=22.05, \underline{p}<.01$, partial $\eta^{2}$ $=.65$. The main effects were qualified by a significant interaction, $\underline{F}(4,48)=10.76, \underline{p}<$ .01 , partial $\eta^{2}=.47$, reflecting the Tutored Training dyads' superior performance on all three inference types. The solution correctness results (see Table 1) matched the dialog

analysis results: while in both the basic and the instructed training conditions only $1 / 3$ of the dyads solved the training case correctly, 7 out of 9 Tutored Training dyads did so (Fisher's exact test, $\mathrm{p}=.12$ ).

Insert Table 1 about here

\subsection{Frequency of inference drawing (Testing phase)}


During the testing phase, all dyads collaborated on the murder-mystery case without receiving any specific guidance. Depending on the kind of training they had received earlier, substantial differences could be observed in the number of inferences dyads drew while solving the murder-mystery. Means and standard deviations are given in Table 2.

Insert Table 2 about here

As expected, the Control dyads showed the lowest frequency of inference drawing overall; they also exhibited most clearly the expected pattern of drawing collaborative < individual $<$ shared inferences, while Tutored Training dyads showed high frequencies of inference drawing for all three inference types (see Figure 4). A two-way mixed model ANOVA with frequency of inference drawing frequency as the dependent variable confirmed that, overall, kind of training had a positive effect on the number of inferences collaborators drew during testing, $\underline{\mathrm{F}}(3,32)=6.03, \underline{\mathrm{p}}<.01$, partial $\eta^{2}=.36$. Planned comparisons of the four conditions further confirmed that trained dyads performed better than those in the Control condition, $\underline{\mathrm{t}}(35)=2.74, \underline{\mathrm{p}}=.01$, Cohen's $\underline{\mathrm{d}}=0.65$. This finding supports Hypothesis 1a, namely that trained dyads would outperform untrained dyads. There was no significant difference in performance between the Basic and Instructed Training dyads, $\underline{\mathrm{t}}(35)=1.12$, $\underline{\mathrm{ns}}$; thus, there was no support for Hypothesis $1 \mathrm{~b}$, namely that direct strategy instruction would increase the effectiveness of basis training. Finally, the Tutored Training dyads did perform better than those in the other two training conditions, $\underline{\mathrm{t}}(35)=3.06, \underline{\mathrm{p}}<.01$, Cohen's $\underline{\mathrm{d}}=0.72$. Thus, the findings support Hypothesis $1 \mathrm{c}$, namely that instructed training in combination with adaptive tutoring would be the most effective kind of training.

Insert Figure 4 about here

Regarding the within subjects factor Inference Type, the expected pattern of students drawing collaborative < individual < shared inferences emerged in nearly all conditions. There was a significant main effect, $\underline{\mathrm{F}}(2,64)=9.55, \underline{\mathrm{p}}<.01$, partial $\eta^{2}=.23$. However, the planned contrasts did not confirm a significant difference in inference drawing frequency between collaborative and individual inferences, $\underline{F}(1,32)=1.13$, $\underline{\mathrm{ns}}$; but they did confirm that collaborative and individual inferences together were less likely to be drawn than shared inferences, $\underline{F}(1,32)=22.37, \underline{p}<.01$, partial $\eta^{2}=.41$. Thus, Hypothesis $2 \mathrm{a}$ was not supported, but Hypothesis $2 \mathrm{~b}$ was. Further, the interaction between kind of training and inference type was not significant, $\underline{F}(6,64)=.79$, $\underline{\mathrm{ns}}$, indicating that the training's effectiveness was not limited to collaborative inferences (as implied by Hypothesis 2c), but generally enhanced the frequency with which students drew inferences.

\subsection{Frequency of information pooling and inference-drawing efficiency}

The frequency of information pooling was high across conditions, though it was even higher for shared information $(\underline{\mathrm{M}}=0.98, \underline{\mathrm{SD}}=0.06)$ than for unshared information 
located within the same person $(\underline{\mathrm{M}}=0.89, \underline{\mathrm{SD}}=0.14)$ or distributed between persons $(\underline{\mathrm{M}}$ $=0.89, \underline{\mathrm{SD}}=0.13$ ). Accordingly, a two-way mixed model ANOVA with information pooling frequency as the dependent variable confirmed a positive main effect for information distribution, $\underline{\mathrm{F}}(2,64)=7.91, \underline{\mathrm{p}}<.01$, partial $\eta^{2}=.19$, but not for kind of training, $\underline{\mathrm{F}}(3,32)=1.76, \underline{\mathrm{ns}}$; the interaction was also nonsignificant. This finding suggests that training was effective not so much by enhancing the frequency with which students pooled information, but rather by increasing the efficiency with which they transformed information into inferences.

\subsection{Solution correctness (Testing phase)}

The murder-mystery testing case was solved correctly by $2 / 3$ of the dyads. Differences in the distribution of wrong vs. correct solutions between conditions were not statistically significant given the relatively small numbers of observations (Fisher's exact test, $\mathrm{p}=.12$ ). Nevertheless, it is informative to observe that the rate of correct solutions increased from the Control condition to the Tutored Training condition, with the notable exception of the Instructed Training condition (see Table 2).

Insert Table 2 about here

\section{Discussion}

Drawing collaborative inferences is an important mechanism of knowledge coconstruction, because collaborative inferences enable groups to generate new knowledge from members' unique, complementary information. Thus, they facilitate collaborative problem-solving and contribute substantially to the advantage of interactive over individual learning (Chi, 2009). However, the present study, in line with prior ones (Deiglmayr \& Spada, 2010a; Härder, 2003; see also Fraidin, 2004), demonstrated that students often miss the possibility of drawing a solution-relevant inference in collaboration with a partner. Two main reasons for this problem are that students may not see the relevance of informing their partner about information they cannot yet make sense of themselves, and that students may not pay sufficient attention to the integration of new information they learn from their partner during discussion (Deiglmayr \& Spada, 2010a). The present study showed that these difficulties can be overcome by appropriate training. Solving a carefully designed training case followed by a collaborative reflection phase (as in the Basic Training condition) substantially improved students' ability to draw collaborative inferences during transfer. This finding is in line with research on "learning-by-doing" showing that students are able to acquire strategic knowledge implicitly from solving carefully designed tasks (Schank et al., 1999) and with research on group processing showing that the transferability of such knowledge can be enhanced by mutual feedback and collaborative reflection (Johnson \& Johnson, 1993). However, the present study was not designed to tease apart the effects of case-based training from the effects of collaborative reflection.

Explicitly informing students about helpful collaboration strategies provided no additional benefit over reflected case-based training (as in the Instructed Training 
condition), at least as long as strategy application was not facilitated by adaptive tutoring. Unexpectedly, Instructed Training dyads solved the testing case much less frequently than dyads in any other condition, indicating the possibility that direct strategy instruction may not only have been unhelpful but may even have had negative side-effects. For example, direct strategy instruction may have led to an illusion of knowing resulting in less effort being invested in collaborative reflection during the group processing phase (Schworm \& Renkl, 2006). This may have caused a misunderstanding of the goals of collaborative information-processing. For example, students may have focused on drawing as many inferences as possible, but neglected to integrate them into a coherent picture from which the correct solution could be developed. Finally, near optimal performance was reached during testing if reflected case-based training had been supported by explicit strategy instruction and adaptive tutoring scaffolding strategy application in the training phase.

Practicing strategy application with adaptive support from the inference tutoring tool, rather than having to apply the strategy instruction all by themselves may have allowed students to acquire strategy knowledge in a more compiled, procedural format that they could apply more flexibly during the testing phase (De Jong \& FergusonHessler, 1996). One reason for this may have been that the tool facilitated strategy application, because it relieved students' working memory by reminding them to take up new information and to look for connections between new and old information (cf. Anderson \& Schunn, 2000). By providing missing information prompts and hints, the tool also provided additional opportunities for students to practice, and thus to automate, the drawing of inferences. With increasing practice, the tool's feedback probably also led to a more direct coupling of observations (e.g., "this information is new for me") and actions (e.g., starting a memory search for complementary information), decreasing the amount of effortful, top-down information-processing necessary for monitoring strategy application (Taatgen, Huss, \& Anderson, 2008).

Finally, it can be assumed that the tutoring tool helped students to better understand the strategies they were being asked to apply. For example, the tutor's prompts (e.g., for taking up new information during discussion) provided students with additional examples of the conditions for strategy application. In this way, the tool may have facilitated students' acquisition of conditional strategy knowledge and thus their later self-regulation of strategy application (Butler \& Winne, 1995). It is possible that adaptive tutoring could lead to the acquisition of applicable strategy knowledge without any prior explicit strategy instruction; however, the data of the present study are not sufficient to explore this question.

\subsection{Limitations of the study}

There are several limitations to the external validity of the present study. First of all, the study used a relatively artificial task in a laboratory situation in order to allow for the distribution of information in each dyad to be manipulated experimentally. In this way, it enabled an exact assessment of the number of collaborative inferences that were drawn and, more importantly, also of the number of collaborative inferences that could have been drawn but were not. Besides the distribution of information between students, additional factors would be of relevance in more authentic collaboration settings, such as 
the classroom, which were not addressed in the present study. One of the most important of these factors probably is the history of the group. While in the present study ad-hoc groups were used, students in a classroom will typically have a history of prior collaboration and grown interpersonal relationships. A history of prior collaboration on similar problems, for example, may lead to the differentiation of expert roles as well as to the emergence of metaknowledge on who is an expert on what kinds of topics (i.e., a transactive memory system; Wegner, 1986). Over time, groups may develop increasing specialisation, thus increasing the need for collaborative inferences bridging members' areas of expertise; but they will also develop an improved transactive memory system facilitating coordination among group members and thus the drawing of collaborative inferences (cf. Michinov \& Michinov, 2009). Further, positive interpersonal feelings like trust or helpfulness may facilitate the pooling of unshared information, and thus the drawing of collaborative inferences, while distrust or disliking of collaboration partners may lead students to withhold information, and thus may hamper the drawing of collaborative inferences (cf. Wittenbaum et al., 2004).

On the other hand, informal observations of students participating in our experiment suggest that the drawing of a collaborative inference is experienced as a particularly rewarding and successful moment of collaboration and thus might have positive social side-effects (cf. Tolmie et al., 2010). Additional research is needed to explore collaborative inferences in larger groups, in more heterogeneous groups, and more complex task domains. For example, all-male or mixed-gender groups might display processes not observed in our all-female dyads (cf. Webb, 1989), in particular in computer-mediated collaboration where males tend to be more active than females (Prinsen, Volman, \& Terwel, 2007). Finally, while our experiment did demonstrate transfer of collaboration skills from a training case to a testing case from a different problem domain, it would be interesting to study whether students could transfer the collaboration skills acquired in the laboratory to more authentic problem-solving situations.

\subsection{Educational implications}

Despite the limitations of the present study, some recommendations can be deduced for educational practice. First, the more group members bring non-overlapping prior knowledge, ideas, or perspectives into the collaboration, the more important collaborative inferences will be for co-constructing new knowledge and solving a joint problem. For example, instructional methods that create expert roles may ensure that students are motivated to collaborate with one another to solve a joint problem, but will also make it more difficult for students to draw solution-relevant inferences bridging different areas of expertise. Second, drawing collaborative inferences requires more than simply pooling information, for example, by mutually explaining one's understanding of the problem. Knowledge co-construction by collaborative inference-drawing is a creative process requiring a more playful, explorative handling of information and ideas (cf. Mercer, 1996). This includes the courage to offer tentative ideas for discussion that may or may not be relevant for finding a solution, and the active take-up and integration of partners' contributions during discussion. These processes might best be supported in an environment encouraging and providing enough time for explorative, divergent thinking. 
Finally, regarding effective training interventions, our results are in line with those from the tutoring literature (e.g., VanLehn et al., 2007) in showing that instruction often is most effective if it is based on careful analyses of the cognitive and communicative processes underlying the targeted skill (i.e. the specific demands of drawing collaborative inferences), is provided in an adaptive fashion that does not overburden students' working memory, and enables students to practice successful strategy application.

\section{Acknowledgments}

The work reported on in this manuscript was funded by the Virtual Graduate School "Knowledge Acquisition and Knowledge Exchange with New Media" of the German Science Foundation. The authors would further like to Kurt VanLehn for inspiring discussions about the design of the inference tutoring tool, and Eva Zerpies for her help with data collection and analysis.

\section{References}

Anderson, J. R., Corbett, A. T., Koedinger, K., \& Pelletier, R. (1995). Cognitive tutors: Lessons learned. The Journal of Learning Sciences, 4, 167-207.

Anderson, J. R., \& Schunn, C. D. (2000). Implications of the ACT-R learning theory: No magic bullets. In R. Glaser (Ed.), Advances in instructional psychology: Educational design and cognitive science (Vol. 5, pp. 1-34). Mahwah, NJ: Erlbaum.

Aronson, E., Balney, N., Sikes, J., \& Snapp, M. (1987). The jigsaw classroom. Beverly Hills, CA: Sage.

Black, A., Freeman, P., \& Johnson-Laird, P. (1986). Plausibility and the comprehension of text. British Journal of Psychology, 77, 51-62.

Bodemer, D., Plötzner, R., Feuerlein, I., \& Spada, H. (2004). The active integration of information during learning with dynamic and interactive visualizations. Learning and Instruction, 14(3), 325-341.

Butler, D., \& Winne, P. (1995). Feedback and self-regulated learning: A theoretical synthesis. Review of Educational Research, 65, 245-81.

Chi, M. T. H. (2009). Active-constructive-interactive: A conceptual framework for differentiating learning activities. Topics in Cognitive Science, 1(1), 73-105.

Collins, A., \& Michalski, R. (1989). The logic of plausible reasoning: A core theory. Cognitive Science, 13, 1-49.

Cohen, E. (1994). Restructuring the classroom: Conditions for productive small groups. Review of Educational Research, 64, 1-35.

Dansereau, D. F., Collins, K. W., McDonald, B. A., Holley, C. D., Garland, J. C., Diekhoff, G., \& Evans, S. H. (1979). Development and evaluation of a learning strategy program. Journal of Educational Psychology, 71, 64-73.

Deiglmayr, A., \& Spada, H., (2010a). Collaborative problem-solving with distributed information: The role of inferences from interdependent information. Group Processes and Intergroup Relations, 13(3), 361-378 
Deiglmayr, A., \& Spada, H. (2010b). Developing adaptive collaboration support: The example of an effective training for collaborative inferences. Educational Psychology Review, 22(1), 103-113.

De Jong, T., \& Ferguson-Hessler, M. (1996). Types and qualities of knowledge. Educational Psychologist, 31(2), 105-113.

Dochy, F., Segers, M., Van den Bossche, P., \& Gijbels, D. (2003). Effects of problem-based learning: A meta-analysis. Learning and Instruction, 13, 533-568.

Fraidin, S. N. (2004). When is one head better than two? Interdependent information in group decision making. Organizational Behavior and Human Decision Processes, 93, 102-113.

Härder, J. (2003). Wissenskommunikation mit Desktop-Videokonferenzen. [Knowledge communication with desktop-videoconferencing systems]. Doctoral dissertation, Albert-Ludwigs-University Freiburg, Germany. Retrieved May 13, 2005, from http://www.freidok.uni-freiburg.de/volltexte/1157

Johnson, D. W., \& Johnson, R. T. (1993). Cooperative learning and feedback in technology-based instruction. In J. V. Dempsey \& G. C. Sales (Eds.), Interactive instruction and feedback (pp. 133-157). Englewood Cliffs, NJ: Educational Technology.

Kaartinen, S., \& Kumpulainen, K. (2002). Collaborative inquiry and the construction of explanations in the learning of science. Learning and Instruction, 12, 189212.

Kintsch, W. (1998). Comprehension: A paradigm for cognition. New York: Cambridge University Press.

Kneser, C., \& Ploetzner, R. (2001). Collaboration on the basis of complementary domain knowledge: Observed dialogue structures and their relation to learning success. Learning and Instruction, 11, 53-83.

Mercer, N. (1996). The quality of talk in children's collaborative activity in the classroom. Learning and Instruction, 6(4), 359-377.

Michinov, N., \& Michinov E. (2009). Investigating the relationship between transactive memory and performance in collaborative learning. Learning and Instruction, $\underline{19}(1), 43-54$.

Pennington, N., \& Hastie, R. (1993). Reasoning in explanation-based decision making. Cognition, 49, 123-163.

Prinsen, F. R., Volman, M. L. L., \& Terwel, J. (2007). Gender-related differences in computer-mediated communication and computer-supported collaborative learning. Journal of Computer Assisted Learning, 23, 393-409.

Rummel, N., \& Spada, H. (2005). Learning to collaborate: An instructional approach to promoting problem-solving in computer-mediated settings. The Journal of the Learning Sciences, 14, 201-241.

Schank, R. C., Berman, T. R., \& Macpherson, K. A. (1999). Learning by doing. In C. M. Reigeluth (Ed.), Instructional-design theories and models (pp. 160-181). Mahwah, NJ: Erlbaum.

Schnotz, W., \& Bannert, M. (2003). Construction and interference in learning from multiple representation. Learning and Instruction, 13, 141-156.

Schworm, S., \& Renkl, A. (2006). Computer-supported example-based learning: When instructional explanations reduce self-explanations. Computers \& Education, 46, 426-445. 
Stasser, G., \& Titus, W. (1985). Pooling of unshared information in group decision making: Biased information sampling during discussion. Journal of Personality and Social Psychology, 48, 1467-1478.

Star, J. R., \& Rittle-Johnson, B. (2008a). Flexibility in problem solving: The case of equation solving. Learning and Instruction, 18, 565-579.

Strøms $\varnothing$, H., Bråten, I., \& Britt, M.A. (2010). Reading multiple texts about climate change: The relationship between memory for sources and text comprehension. Learning and Instruction, 20, 192-204.

Taatgen, N. A., Huss, D., \& Anderson, J. R. (2008). The acquisition of robust and flexible cognitive skills. Journal of Experimental Psychology: General, 137(3), 548-565.

Tolmie, A. K., Topping, K., Christie, D., Donaldson, C., Howe, C., Jessiman, E., Livingston, K., \& Thurston, A. (2010). Social effects of collaborative learning in primary schools. Learning and Instruction, 20, 177-191.

VanLehn, K., Graesser, A. C., Jackson, G. T., Jordan, P., Olney, A., \& Rose, C. P. (2007). When are tutorial dialogues more effective than reading? Cognitive Science, 31, 3-62.

Webb, N. M (1989) Peer interaction and learning in small groups. International Journal of Educational Research, 13(1), 21-39.

Webb, N. M., \& Palincsar, A. S. (1996). Group processes in the classroom. In D. Berliner \& R. Calfee (Eds.), Handbook of educational psychology (pp. 841-873). New York: Macmillan.

Wegner, D. (1986). Transactive memory: A contemporary analysis of the group mind. In B. Mullen \& G. R. Goethals (Ed.), Theories of group behavior (pp. 185-208). New York: Springer.

Wittenbaum, G., Hollingshead, A., \& Botero, I. (2004). From cooperative to motivated information sharing in groups: Moving beyond the hidden profile paradigm. Communication Monographs, 71, 286-310.

Zohar, A., \& Peled, B. (2008). The effects of explicit teaching of metastrategic knowledge on low- and high-achieving students. Learning and Instruction, 18, 337-353. 
Table 1. Means (and $\underline{\mathrm{SD}}$ ) of frequencies of inference drawing while solving the training case as a function of training condition and inference type, and number of dyads with correct solution in each training condition

\begin{tabular}{lllll}
\hline & $\begin{array}{l}\text { Control } \\
(\underline{\mathrm{n}}=9 \text { dyads })\end{array}$ & $\begin{array}{l}\text { Basic training } \\
(\underline{\mathrm{n}}=9 \text { dyads })\end{array}$ & $\begin{array}{l}\text { Instructed } \\
\text { training } \\
(\underline{\mathrm{n}}=9 \text { dyads })\end{array}$ & $\begin{array}{l}\text { Tutored } \\
\text { training } \\
(\underline{\mathrm{n}}=9 \text { dyads })\end{array}$ \\
\cline { 2 - 5 }$(\underline{\mathrm{SD}})$ & $\underline{\mathrm{SD}})$ & $\underline{\mathrm{M}})$ \\
\hline Collaborative inferences & -- & $0.42(0.25)$ & $0.31(0.20)$ & $0.98(0.07)$ \\
Individual inferences & -- & $0.72(0.23)$ & $0.75(0.28)$ & $0.97(0.08)$ \\
Shared inferences & -- & $0.96(0.11)$ & $1.00(0.00)$ & $1.00(0.00)$ \\
\hline Number of dyads with correct solution & -- & 3 & 3 & 7 \\
\hline
\end{tabular}

Table 2. Means (and SD) of frequencies of inference drawing while solving the testing case as a function of training condition and inference type, and number of dyads with correct solution in each training condition

\begin{tabular}{lllll}
\hline & $\begin{array}{l}\text { Control } \\
(\underline{\mathrm{n}}=9 \text { dyads })\end{array}$ & $\begin{array}{l}\text { Basic training } \\
(\underline{\mathrm{n}}=9 \text { dyads })\end{array}$ & $\begin{array}{l}\text { Instructed } \\
\text { training } \\
(\underline{\mathrm{n}}=9 \text { dyads })\end{array}$ & $\begin{array}{l}\text { Tutored } \\
\text { training } \\
(\underline{\mathrm{n}}=9 \text { dyads })\end{array}$ \\
\cline { 2 - 5 }$(\underline{\mathrm{SD}})$ & $\underline{\mathrm{SD}})$ & $\underline{\mathrm{M}})$ \\
\hline Collaborative inferences & $0.56(0.27)$ & $0.72(0.23)$ & $0.69(0.24)$ & $0.89(0.18)$ \\
Individual inferences & $0.72(0.15)$ & $0.81(0.24)$ & $0.64(0.28)$ & $0.92(0.13)$ \\
Shared inferences & $0.83(0.22)$ & $0.92(0.18)$ & $0.92(0.18)$ & $1.00(0.00)$ \\
\hline Number of dyads with correct solution & 6 & 7 & 3 & 8 \\
\hline
\end{tabular}




\begin{tabular}{|c|c|c|c|c|}
\hline $\begin{array}{l}\text { Information } \\
\text { distribution }\end{array}$ & Student A & Student B & Inference type & \\
\hline $\begin{array}{l}\text { unshared } \\
\text { distributed }\end{array}$ & & & $\begin{array}{c}\text { collaborative } \\
\text { ב }\end{array}$ & $\Rightarrow$ \\
\hline $\begin{array}{l}\text { unshared } \\
\text { undistributed }\end{array}$ & & & individual & \\
\hline shared & & & shared & \\
\hline
\end{tabular}

Fig. 1: Visualization of collaborative, individual, and shared inferences.

\begin{tabular}{|c|c|c|c|c|}
\hline & $\begin{array}{l}\text { Control (no training) } \\
(\underline{n}=9 \text { dyads })\end{array}$ & $\begin{array}{l}\text { Basic Training } \\
(\underline{n}=9 \text { dyads })\end{array}$ & $\begin{array}{l}\text { Instructed Training } \\
(\underline{n}=9 \text { dyads })\end{array}$ & $\begin{array}{l}\text { Tutored Training } \\
(\underline{\mathrm{n}}=9 \text { dyads })\end{array}$ \\
\hline & \multicolumn{4}{|l|}{ Introduction } \\
\hline \multirow{5}{*}{ 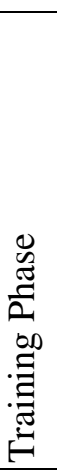 } & -- & & \multicolumn{2}{|c|}{ explicit strategy instruction (5 $\mathrm{min})$} \\
\hline & \multirow[t]{4}{*}{--} & \multicolumn{3}{|c|}{$\begin{array}{l}\text { training case (medical-diagnosis problem) } \\
\text { individual reading phase ( } 20 \mathrm{~min})\end{array}$} \\
\hline & & \multicolumn{2}{|c|}{ discussion with partner (25 min) } & $\begin{array}{l}\text {... with guidance from } \\
\text { inference tutoring tool }\end{array}$ \\
\hline & & \multicolumn{3}{|c|}{ written justification (prepared collaboratively) (5 min) } \\
\hline & & \multicolumn{3}{|c|}{$\begin{array}{l}\text { collaborative reflection phase }(10 \mathrm{~min}) \\
\text { read and discuss model solution } \\
\text { reflect past and upcoming collaboration }\end{array}$} \\
\hline 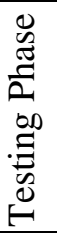 & \multicolumn{4}{|c|}{$\begin{array}{l}\text { testing case (murder-mystery problem) } \\
\text { individual reading phase }(30 \mathrm{~min}) \\
\text { discussion with partner }(30 \mathrm{~min}) \\
\text { written justification (prepared collaboratively) }(10 \mathrm{~min})\end{array}$} \\
\hline
\end{tabular}

Fig. 2: Experimental procedure in the four conditions. 


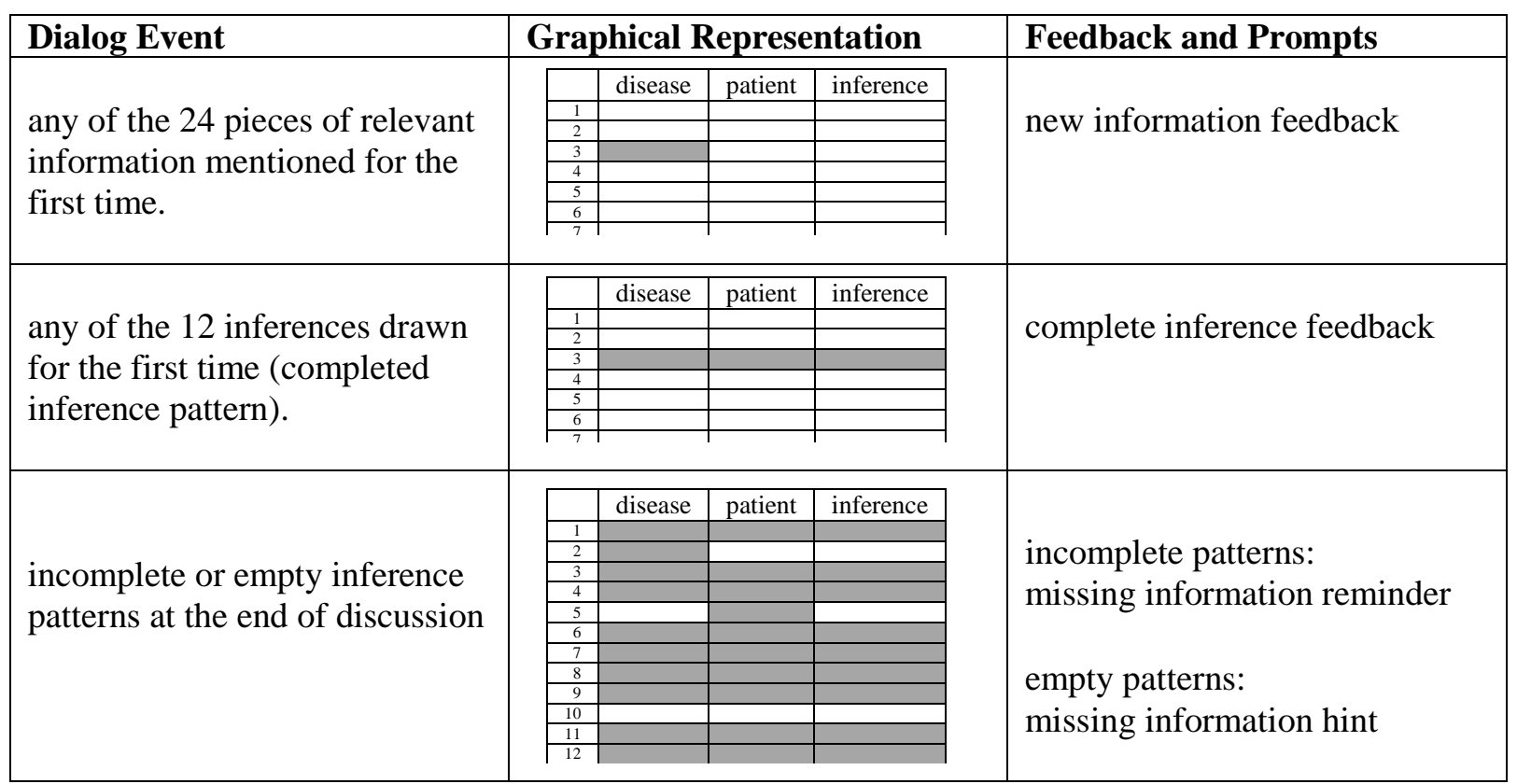

Fig. 3. Feedback and prompts sent through the inference tutoring tool based on observed dialog content (see text for examples). 


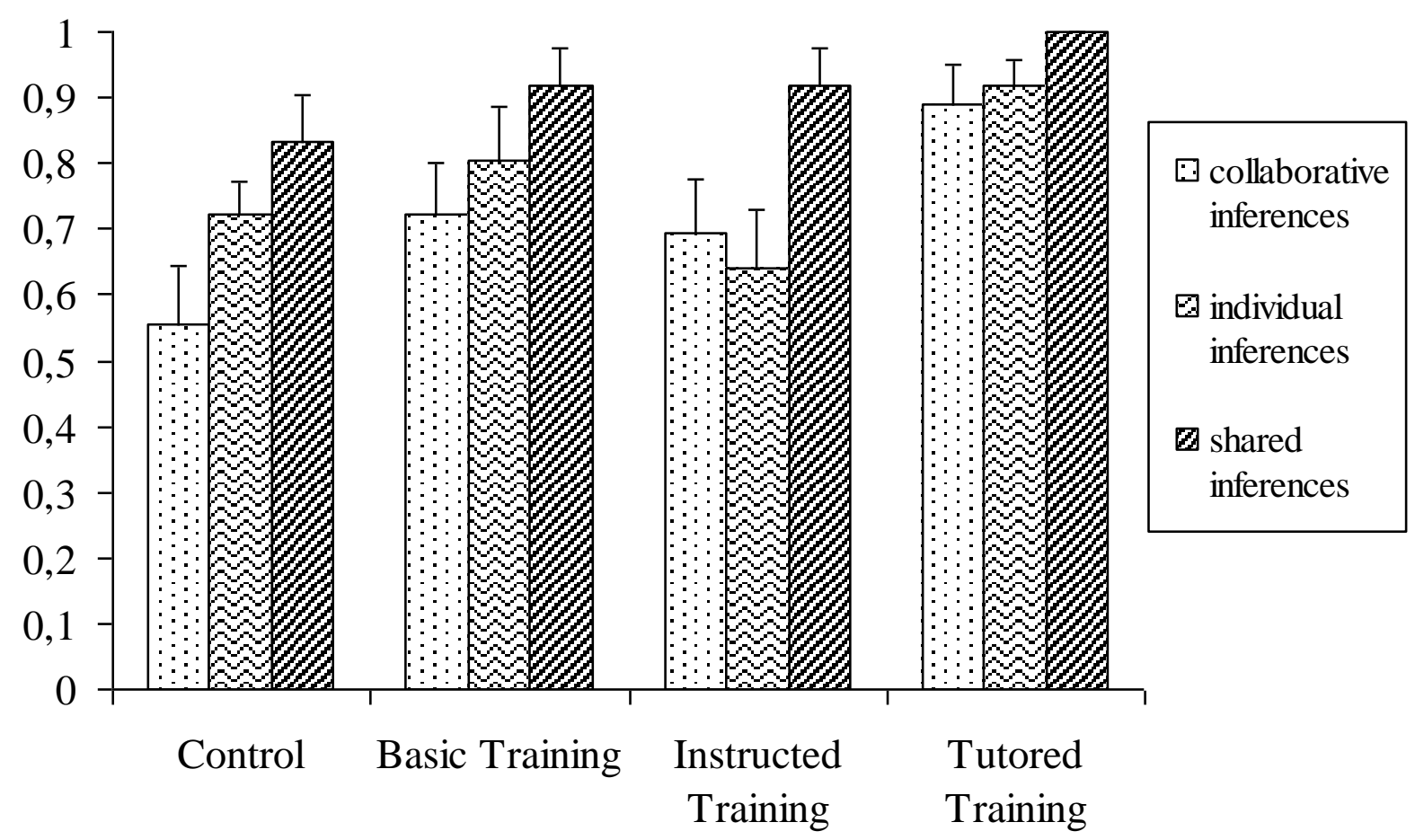

Fig. 4. Testing Phase: Inference drawing frequency (means and standard errors) during testing on the murder-mystery problem (Tutored Training dyads: without inference tutoring tool). 